\title{
A note on the qualitative behavior of some nonlinear local improper conformable differential equations
}

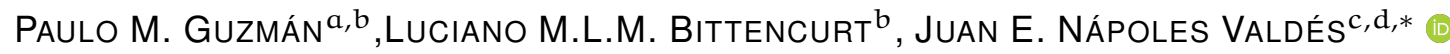 \\ a Universidad Nacional del Nordeste, Facultad de Ciencias Agrarias Corrientes Capital, \\ 3400, Argentina \\ b Universidad Nacional del Nordeste, Facultad de Ciencias Exactas y Naturales y \\ Agrimensura Corrientes Capital, 3400, Argentina \\ c UNNE, FaCENA, Ave. Libertad 5450, Corrientes 3400, Argentina \\ d UTN-FRRE, French 414, Resistencia, Chaco 3500, Argentina
}

- Received: 06.11.2020 • Accepted: 18.12.2020 • Published Online:30.12.2020

\begin{abstract}
In this paper, we present two qualitative results concerning the solutions of nonlinear generalized differential equations, with a local derivative defined by the authors in previous works. The first result covers the boundedness of solutions while the second one discusses when all the solutions are in $\mathrm{L}^{2}$.
\end{abstract}

Keywords: Bounded, $\mathrm{L}^{2}$-solutions, square-integrable, asymptotic behavior. 2010 MSC: 34C10, 34K11.

\section{Introduction \& Preliminaries}

The nature of numerous systems sorts that they can be more accurately modeled utilizing fractional differential equations (FDEs). The fractional calculus have found applications in various fields of science and engineering like electrochemistry, viscoelasticity, mechanics, control theory, diffusion processes, heat conduction, electricity, chaos, and fractals(cf. [1, 2, 3, 4]). In the last few decades, research of varied aspects of these equations and his qualitative theory has been paid much attention by many authors (see $[5,6,7,8,9,10,11,12,13,14,15,16,17,18,19]$ and references cited therein). In these research, two qualitative properties that have not been well studied is the case of the boundedness of the solutions and which are square integrable.

\footnotetext{
*Corresponding author: jnapoles@exa.unne.edu.ar
}

(C) 2020 SABA. All Rights Reserved. 
Although local operators began to be used since the 1960s, it was not until 2014 (see [20] and [21] for example), when defined fractional operators appear in terms of a certain incremental cocient, unlike the classics, which are defined using a certain integral operator. The multiplicity of applications of fractional operators, local and global, has led to the appearance of new differential and integral operators, causing that there is no single fractional derivative operator, or at least one unanimously accepted definition, see [22] for a quite complete classification of the most used operators.

It is noteworthy that, in general, they are centred on equations with the classical "global" fractional derivatives and qualitative research is almost non-existent, using local fractional derivatives (see [14, 15, 16] and [18] for attempts in that direction, the first three in a global sense and the last two local ones). In [23] it is proposed the definition of an improper conformable local derivative (originally named non-conformable), various applications and extensions of this derivative can be consulted at [24, 25, 26, 27, 28, 29].

Definition 1.1. Let $f:[0,+\infty) \rightarrow \mathbb{R}$. The $\mathcal{N}$-derivative of $f$ of order $\delta$ is defined by

$$
\mathcal{N}_{1}^{\delta} f(\mathfrak{r})=\lim _{\varepsilon \rightarrow 0} \frac{f\left(\mathfrak{r}+\varepsilon e^{\mathfrak{r}^{-\delta}}\right)-f(\mathfrak{r})}{\varepsilon}, \text { for all } \mathfrak{r}>0, \delta \in(0,1) .
$$

Moreover, If $f$ is $\delta$-differentiable in some $(0, a)$, and $\lim _{\mathfrak{r} \rightarrow 0^{+}} \mathcal{N}_{1}^{(\delta)} f(\mathfrak{r})$ exists, then we have $\mathcal{N}_{1}^{(\delta)} f(0)=\lim _{\mathfrak{r} \rightarrow 0^{+}} \mathcal{N}_{1}^{(\delta)} f(\mathfrak{r})$.

The conformable term which is sometimes used in local fractional derivatives, may or may not be appropriate here, since this was initially referred to as a conformable fractional derivative $D^{\delta} f(\mathfrak{r})$, when $\delta \rightarrow 1$ satisfies $D^{\delta} f(\mathfrak{r}) \rightarrow f^{\prime}(\mathfrak{r})$; i.e., when $\delta \rightarrow 1$, $D^{\delta} f(\mathfrak{r})$ preserves the angle of the tangent line to the curve, while in our definition, this angle is not conserved. However, there is a detail that we want to point out, our derivative is conformable "at infinity", that is, when t tends to infinity, our derivative coincides with the classical derivative, which is of vital importance in the qualitative theory.

Also, the following properties will be used throughout the work:

a) $\mathcal{N}_{1}^{\delta}(f g)(\mathfrak{r})=f \mathcal{N}_{1}^{\delta}(g)(\mathfrak{r})+g \mathcal{N}_{1}^{\delta}(f)(\mathfrak{r})$

b) If $f$ is differentiable then $\mathcal{N}_{1}^{\delta}(\mathfrak{f})(\mathfrak{r})=e^{\mathfrak{r}^{-\delta}} f^{\prime}(\mathfrak{r})$

c) $\mathcal{N}_{1}^{\delta}(f \circ g)(\mathfrak{r})=\mathcal{N}_{1}^{\delta} f^{\prime}(g(\mathfrak{r}))=f^{\prime}(g(\mathfrak{r})) \mathcal{N}_{1}^{\delta} g(\mathfrak{r})$.

Throughout the paper we will denote by $\mathfrak{I}:=\left[\mathfrak{r}_{0},+\infty\right), \mathbb{R}:=\mathfrak{I} \times \mathbb{R}$ and $K:=\mathfrak{I} \times \mathbb{R}^{2}$ and $\mathfrak{r}_{0}>0$.

In this note, based on the above definition of derivative, we consider the forced improper conformable FDEs differential equations of order $\delta$ with damping:

$$
\mathcal{N}_{1}^{\delta}\left[p(\mathfrak{r}) \mathcal{N}_{1}^{\delta} \varkappa(\mathfrak{r})\right]+\mathrm{f}(\mathfrak{r}, \varkappa(\mathfrak{r})) \mathcal{N}_{1}^{\delta} \varkappa(\mathfrak{r})+g(\mathfrak{r}, \varkappa(\mathfrak{r}))=e(\mathfrak{r}),
$$

for $\mathfrak{r} \in \mathfrak{I}$, and $0<\delta<1$, under the following assumptions:

i) $p(\mathfrak{r}), e(\mathfrak{r})$ are continuous functions on $\mathfrak{I}$ with $0<p \leqslant p(\mathfrak{r})<+\infty$ and $e(\mathfrak{r})$ is square-integrable on $\mathfrak{I}$.

ii) $\mathrm{f} \in \mathrm{C}\left(\mathfrak{I}, \mathbb{R}_{+}\right)$with $0<\mathrm{f}_{0} \leqslant \mathrm{f}(\mathfrak{r}, \varkappa)$, and $\mathrm{g} \in \mathrm{C}^{(0, \delta)}(\mathfrak{I}, \mathbb{R})$ such that $\int_{0}^{ \pm \infty} \mathrm{g}(\mathfrak{r}, \varkappa) \mathrm{d} \varkappa=$ $\pm \infty$ uniformly in $\mathfrak{r}$ and $\varkappa \frac{\partial g(\mathfrak{r}, \varkappa)}{\partial \mathfrak{r}} \leqslant 0$. 
We will establish sufficient conditions for boundedness and $\mathrm{L}^{2}$ properties of solutions of Eq. (1.1). Our approach differs greatly from those of the previous research as all they built energy or Liapunov functions, thus our results are obtained by techniques very different from those previously reported.

The solutions of Eq. (1.1) are bounded if there exists a constant $K>0$ such that $|\varkappa(\mathfrak{r})|<\mathrm{K}$ for all $\mathfrak{r} \geqslant \mathrm{T}>0$ for some $\mathrm{T}$. By an $L^{2}$-solution, we mean a solution of Eq. (1) such that $\int_{\mathfrak{r}_{0}}^{\infty} \varkappa^{2}(\mathfrak{r}) \mathrm{d} \mathfrak{r}<\infty$.

The classical Liénard equation $\varkappa^{\prime \prime}+f(\varkappa) \varkappa^{\prime}+g(\varkappa)=0$, and its equivalent system (with $F(\varkappa)=\int_{0}^{\varkappa} f(r) d r$ )

$$
\left\{\begin{array}{l}
\varkappa^{\prime}=y-F(\varkappa) \\
y^{\prime}=-g(\varkappa)
\end{array}\right.
$$

shows up as a rearranged model in numerous areas in science and engineering. It has been studied extensively during the first half of the twentieth century as it very well may be utilized to model simple pendulums or oscillating circuits, for this situation, $f$ and $g$ represent the friction and acceleration terms. The Liénard equation is frequently taken as the typical example of a nonlinear self-excited vibration problem, it can likewise be utilized to model resistor-inductor-capacitor circuits with nonlinear circuit components, and model certain mechanical systems with nonlinear damping, stiffness, or restoring force. In addition, some nonlinear evolution equations (such as the Burgers-Kortewegde Vries equation) which emerge from different physical phenomena can also be turned into the Liénard equation. One of the main models where this equation shows up was presented by Van der Pol, he considered the equation

$$
\varkappa^{\prime \prime}+\mu\left(\varkappa^{2}-1\right) \varkappa^{\prime}+\varkappa=0,
$$

for modeling the oscillations of a triode vacuum tube. In this way, the investigation of these equations is of actual importance in physic.

On the other hand, the investigation of these equations has prompted research that has affected different mathematical areas, to have a more complete idea of their significance for nonlinear analysis in specifically, and for mathematics in general, it very well may be counseled in $[30,31]$.

Notwithstanding, in the non-integer case, the consideration that has gotten in these equations is scarce and negligible.

In [32], the authors considered the following system:

$$
\left\{\begin{array}{c}
{ }_{0}^{C} D_{\mathfrak{r}}^{\delta} \varkappa(\mathfrak{r})=y(\mathfrak{r})-F(\varkappa(\mathfrak{r})) \\
{ }_{0}^{C} D_{\mathfrak{r}}^{\delta} y(\mathfrak{r})=-g(\varkappa(\mathfrak{r}))
\end{array}\right.
$$

as a natural generalization of the classical Liénard system, using the Caputo fractional derivative, with $F(\varkappa)$ as above, and $f$ and $g$ are continuous functions such that $\mathrm{f}: \mathbb{R} \rightarrow \mathbb{R}_{+}$, and $\mathrm{g}: \mathbb{R} \rightarrow \mathbb{R}$ with $\varkappa \mathrm{g}(\varkappa)>0$ for $\varkappa \neq 0$. The above system is equivalent to the Liénard fractional (global) equation ${ }_{0}^{C} D_{\mathfrak{r}}^{2 \delta} \varkappa(\mathfrak{r})+{ }_{0}^{C} D_{\mathfrak{r}}^{\delta}[F(\varkappa(\mathfrak{r}))]+g(\varkappa(\mathfrak{r}))$. It is then natural to study generalizations of the Liénard equation using local fractional derivatives. 
If in Eq. (1.1) we make $p(\mathfrak{r}) \equiv 1, e(\mathfrak{r}) \equiv 0, f(\mathfrak{r}, \varkappa)=f(\varkappa)$ and $g(\mathfrak{r}, \varkappa)=g(\varkappa)$, it is clear that equation Eq. (1.1) becomes equation

$$
\mathcal{N}_{1}^{\delta}\left[\mathcal{N}_{1}^{\delta} \varkappa(\mathfrak{r})\right]+f(\varkappa(\mathfrak{r})) \mathcal{N}_{1}^{\delta} \varkappa(\mathfrak{r})+g(\varkappa(\mathfrak{r}))=0,
$$

our generalization of the Liénard Equation. So, every qualitative result for Eq. (1.1) produces a qualitative result for Eq. (1.2), the equivalent Liénard fractional equation but in local sense and improper conformable.

\section{Results}

We are now ready to prove a general boundedness theorem.

Theorem 2.1. We assume that conditions $i)$, ii) above hold. Then any solution $\varkappa(\mathfrak{r})$ of Eq. (1.1), as well as its derivative, is bounded as $\mathfrak{r} \rightarrow+\infty$ and $\int_{\mathfrak{r}_{0}}^{\infty} \varkappa^{\prime 2}(\mathfrak{r}) \mathrm{d} \mathfrak{r}<\infty$.

Proof. By standard existence theory, there is a solution of Eq. (1.1) which exists on $\left[\mathfrak{r}_{0}, \mathfrak{r}\right.$ ) for some $\mathrm{T}>\mathfrak{r}_{0}>0$.

Set

$$
\begin{gathered}
\xi_{0}=\int_{\mathfrak{r}_{0}}^{T} e^{-s^{-\delta}} d s, \xi=\int_{\mathfrak{r}_{0}}^{\mathfrak{r}} e^{-s^{-\delta}} d s, P(\xi)=p(\mathfrak{r}), \quad E(\xi)=e(\mathfrak{r}), \\
F(\xi, \mathfrak{X}(\xi))=f(\mathfrak{r}, \varkappa(\mathfrak{r})), \quad G(\xi, \mathfrak{X}(\xi))=g(\mathfrak{r}, \varkappa(\mathfrak{r})),
\end{gathered}
$$

Let $\varkappa(\mathfrak{r})=\mathfrak{X}(\xi)$. Then $\mathcal{N}_{1}^{\delta} \varkappa(\mathfrak{r})=\varkappa^{\prime}(\xi)$ so, Eq. (1.1) is transformed into

$$
\left(P(\xi) \mathfrak{X}^{\prime}(\xi)\right)^{\prime}+F(\xi, \mathfrak{X}(\xi)) \varkappa^{\prime}(\xi)+G(\xi, \mathfrak{X}(\xi))=E(\xi),
$$

Multiply Eq. (2.1) by $\mathfrak{X}^{\prime}$ and then use the integration by parts from $\xi_{0}$ to $\xi$ on the last term of the left hand side of Eq. (2.1) we get

$$
\left\{\begin{array}{l}
p \frac{\left(\varkappa^{\prime}(\xi)\right)^{2}}{2}+\int_{\xi_{0}}^{\xi} \mathrm{F}(z, \mathfrak{X}(z))\left(\mathfrak{X}^{\prime}(z)\right)^{2} \mathrm{~d} z+\int_{\varkappa(\xi}^{\varkappa(\xi)} \mathrm{G}(\xi, \mathfrak{u}) \mathrm{d} u- \\
\quad-\int_{\xi_{0}}^{\xi} \int_{\varkappa\left(\xi_{0}\right)}^{\varkappa\left(\left(\xi_{0}\right)\right.} \frac{\partial \mathrm{G}(z, \mathfrak{u})}{\partial z} \mathrm{~d} u \mathrm{~d} z \\
\quad \leqslant \mathrm{p} \frac{\left(\mathfrak{X}^{\prime}\left(\xi_{0}\right)\right)^{2}}{2}+\int_{\xi_{0}}^{\xi}\left|\mathrm{E}(z) \mathfrak{X}^{\prime}(z)\right| \mathrm{d} z .
\end{array}\right.
$$

Now if $\mathfrak{X}(\xi)$ becomes unbounded then we must have that each term on the L.H.S. of Eq. (2.2) become positive from our assumptions. By the Cauchy-Schwarz inequality for integrals on the R.H.S. of Eq. (2.2), we obtain

$$
\begin{aligned}
& p \frac{\left(\varkappa^{\prime}(\xi)\right)^{2}}{2}+\int_{\xi_{0}}^{\xi} F(z, \mathfrak{X}(z))\left(\mathfrak{X}^{\prime}(z)\right)^{2} \mathrm{~d} z+\int_{\varkappa\left(\xi_{0}\right)}^{\varkappa(\xi)} \mathrm{G}(\xi, u) \mathrm{d} u- \\
& -\int_{\xi_{0}}^{\xi} \int_{\varkappa\left(\xi_{0}\right)}^{\varkappa(\xi)} \frac{\partial \mathrm{G}(z, \mathfrak{u})}{\partial z} \mathrm{~d} u \mathrm{~d} z \\
\leqslant & p \frac{\left(\mathfrak{X}^{\prime}\left(\xi_{0}\right)\right)^{2}}{2}+\left(\int_{\xi_{0}}^{\xi} E^{2}(z) d z\right)^{\frac{1}{2}}\left(\int_{\xi_{0}}^{\xi}\left(\mathfrak{X}^{\prime}(z)\right)^{2} \mathrm{~d} z\right)^{\frac{1}{2}} .
\end{aligned}
$$


Now, let $\mathcal{H}(\mathfrak{r})=\left(\int_{\xi_{0}}^{\xi}\left(\mathfrak{X}^{\prime}(z)\right)^{2} \mathrm{~d} z\right)^{\frac{1}{2}}$. Dividing both sides by $\mathrm{H}(\mathfrak{r})$ yields

$$
\left\{\begin{array}{l}
\mathcal{H}^{-1}(\mathfrak{r})\left[p \frac{\left(\mathfrak{X}^{\prime}(\xi)\right)^{2}}{2}+\int_{\xi_{0}}^{\xi} \mathrm{F}(z, \mathfrak{X}(z))\left(\mathfrak{X}^{\prime}(z)\right)^{2} \mathrm{~d} z+\int_{\varkappa\left(\xi_{0}\right)}^{\varkappa(\xi)} \mathrm{G}(\xi, \mathrm{u}) \mathrm{d} u-\right. \\
\left.-\int_{\xi_{0}}^{\xi} \int_{\varkappa(\xi)}^{\varkappa(\xi)} \frac{\partial \mathrm{G}(z, \mathfrak{u})}{\partial z} \mathrm{~d} u \mathrm{~d} z\right] \\
\leqslant \mathcal{H}^{-1}(\mathfrak{r}) \mathrm{p} \frac{\left(\mathfrak{X}^{\prime}\left(\xi_{0}\right)\right)^{2}}{2}+\left(\int_{\xi_{0}}^{\xi} \mathrm{E}^{2}(z) \mathrm{d} z\right)^{\frac{1}{2}} .
\end{array}\right.
$$

Taking into account the positivity of L.H.S. of Eq. (2.3) if $\mathfrak{X}(\mathfrak{r})$ increase without bound and that term

$$
\mathcal{H}^{-1}(\mathfrak{r}) \mathrm{f}_{0} \int_{\xi_{0}}^{\xi}\left(\mathfrak{X}^{\prime}(z)\right)^{2} \mathrm{~d} z=\mathrm{f}_{0}\left(\int_{\xi_{0}}^{\xi}\left(\mathfrak{X}^{\prime}(z)\right)^{2} \mathrm{~d} z\right)^{\frac{1}{2}}
$$

is bounded by the R.H.S. of Eq. (2.3) we get that $\mathfrak{X}^{\prime}$ is square integrable and is also a bounded after we examine the first term of the L.H.S. of Eq. (2.3). However, the above implies that $|\mathfrak{X}(\xi)|$ must be bounded. Otherwise, the L.H.S. of Eq. (2.3) becomes infinite which is impossible. A standard argument now permits the solution to be extended on all $\mathfrak{r}$ of $\mathfrak{I}$, see e.g., [33] and [34]. The proof is finished.

By imposing more strict conditions on $g(\mathfrak{r}, \varkappa)$ and $p(\mathfrak{r})$, all solutions become $\mathrm{L}^{2}$ solutions. This case is covered by the following Theorem.

Theorem 2.2. Under assumptions of Theorem 2.1. Let $\mathrm{g}(\mathfrak{r}, \varkappa) \varkappa>\mathrm{g}_{0} \varkappa^{2}$ for some positive constant $\mathrm{g}_{0}$, and $0<\mathrm{p}<\mathrm{p}(\mathfrak{r})<\mathrm{P}<+\infty$. Then all the solutions of $E q$. (1) are $L^{2}$-solutions.

Proof. In order to show that $\varkappa^{2} \in \mathrm{L}^{2}[0,+\infty)$, we must first multiply Eq. (2.1) by $\mathfrak{X}$, the integration from $\xi_{0}$ to $\xi$ yields

$$
\begin{aligned}
& \mathfrak{X}(\xi)\left(\mathrm{P}(\xi) \mathfrak{X}^{\prime}(\xi)\right)-\int_{\xi_{0}}^{\xi} \mathrm{P}(z)\left(\mathfrak{X}^{\prime}(z)\right)^{2} \mathrm{~d} z+\int_{\xi_{0}}^{\xi} \mathrm{F}(z, \mathfrak{X}(z)) \mathfrak{X}^{\prime}(z) \mathfrak{X}(z) \mathrm{d} z+ \\
& +\int_{\xi_{0}}^{\xi} \mathrm{G}(z, \mathfrak{X}(z)) \mathfrak{X}(z) \mathrm{d} z \\
= & \mathfrak{X}\left(\xi_{0}\right)\left(\mathrm{P}\left(\xi_{0}\right) \mathfrak{X}^{\prime}\left(\xi_{0}\right)\right)+\int_{\xi_{0}}^{\xi} \mathrm{E}(z) \mathfrak{X}(z) \mathrm{d} z .
\end{aligned}
$$

Next, let $F(\mathfrak{X}(\xi))=\int_{\mathfrak{X}\left(\xi_{0}\right)}^{\mathfrak{X}(\xi)} F\left(\mathfrak{X}^{-1}(\mathfrak{u}), \mathfrak{u}\right)$ udu. So, Eq. (2.4) may be rewritten as

$$
\mathfrak{X}(\xi)\left(P(\xi) \mathfrak{X}^{\prime}(\xi)\right)-P \int_{\xi_{0}}^{\xi}\left(\mathfrak{X}^{\prime}(z)\right)^{2} \mathrm{~d} z+\mathrm{F}(\mathfrak{X}(\xi))+g_{0} \int_{\xi_{0}}^{\xi} \mathfrak{X}^{2}(z) d z \leqslant K,
$$

where

$$
\mathrm{K}=\mathfrak{X}\left(\xi_{0}\right)\left(\mathrm{P}\left(\xi_{0}\right) \mathfrak{X}^{\prime}\left(\xi_{0}\right)\right)+\left|\int_{\xi_{0}}^{\xi} \mathrm{E}(z) \mathfrak{X}(z) \mathrm{d} z\right| .
$$


Notice that the last term is bounded by

$$
\left(\int_{\xi_{0}}^{\xi} E^{2}(z) d z\right)^{\frac{1}{2}}\left(\int_{\xi_{0}}^{\xi}(\mathfrak{X}(z))^{2} d z\right)^{\frac{1}{2}}
$$

due to using the Cauchy-Scwharz inequality. Dividing Eq. (2.5) by $M(\xi)=\left(\int_{\xi_{0}}^{\xi}(\mathfrak{X}(z))^{2} \mathrm{~d} z\right)^{\frac{1}{2}}$ we obtain

$$
\left\{\begin{array}{c}
\mathrm{M}^{-1}(\xi)\left[\mathfrak{X}(\xi)\left(\mathrm{P}(\xi) \mathfrak{X}^{\prime}(\xi)\right)-\mathrm{P} \int_{\xi_{0}}^{\xi}\left(\mathfrak{X}^{\prime}(z)\right)^{2} \mathrm{~d} z+\mathrm{F}(\mathfrak{X}(\xi))\right]+ \\
+\mathrm{g}_{0}\left[\int_{\xi_{0}}^{\xi} \mathfrak{X}^{2}(z) \mathrm{d} z\right]^{\frac{1}{2}} \leqslant \frac{\mathrm{K}}{\mathrm{M}(\xi)},
\end{array}\right.
$$

Since the R.H.S of Eq. (2.6) is bounded and all the terms of the L.H.S are either bounded or positive, the result follows because the L.H.S cannot be unbounded. Here, we shown that $\varkappa$ is square integrable.

Remark 2.3. Under assumptions $0<p \leqslant p(\mathfrak{r})$ and $f(\mathfrak{r}, \varkappa) \geqslant f_{0}>0$ for some positive constants $p$ and $f_{0}$, the class of Eq. (1.1) is not very large, but if this conditions are not fulfilled, we can exhibit equations that have unbounded solutions. For example

$$
\mathcal{N}_{1}^{\delta}\left[e^{-\mathfrak{r}^{-\delta}} \mathcal{N}_{1}^{\delta} \varkappa(\mathfrak{r})\right]-2 \mathcal{N}_{1}^{\delta} \varkappa(\mathfrak{r})+e^{\mathfrak{r}^{-\delta}} \varkappa(\mathfrak{r})=0
$$

has the unbounded solution $\varkappa(\mathfrak{r})=e^{\mathfrak{r}}$.

\section{Conclusion}

In this paper, we deal with the qualitative behavior of the solutions of a differential equation, which involves a derivative defined by the authors in a previous work, using a certain transformation. As you can see, the change of variables used is very important, since it transforms an improper conformable differential equation into an ordinary integer differential equation, whose oscillation criteria can be established using the generalized Riccati Transformation The results obtained can be generalized to investigate the oscillation of differential equations with other local derivatives and with higher orders.

Regarding the technique used in this work, we would like to add a final observation.

Although in recent years the number of researchers and research on the qualitative properties of FDEs has increased, in many of these works a Geometric Transformation like the one used by us in this work is used. However, we must say that this proliferation of results has caused that sometimes the results are not correct (to mention just a few examples we refer the reader to [7], [8], [35], [10], [14], [15], [17], [36], [37], [19], and [38]), all of which use a result of [39], where a Chain Rule is presented for a modification of 
the Fractional Derivative of type Riemann-Liouville, which is incorrect (see [40] who put several counter-examples that prove the incorrectness of said result). In this way, all the results obtained in said works, which use a Geometric Transformation that implicitly uses the Chain Rule, are wrong.

Acknowledgment. The authors wish to thank the reviewers for their suggestions and indications, which have improved the quality of the work.

\section{References}

[1] Kilbas AA, Srivastava HM and Trujillo JJ (2006). "Theory and applications of fractional differential equations". Math. Studies, North-Holland, New York.

[2] Miller KS (1993). "An Introduction to Fractional Calculus and Fractional Differential Equations" J. Wiley and Sons, New York.

[3] Oldham K and Spanier J (1974). "The fractional calculus: Theory and applications of differentiation and integration of arbitrary order". Academic Press, USA.

[4] Podlubny I (1999). "Fractional Differential Equations". Academic Press, USA.

[5] Aguila-Camacho N and Duarte-Mermoud MA (2016). Boundedness of the solutions for certain classes of fractional differential equations with application to adaptive systems. ISA Transactions 60: 82-88. https:/ / doi.org/10.1016/j.isatra.2015.11.013

[6] Aguila-Camacho N, Duarte-Mermoud MA and Gallegos JA (2014). Lyapunov functions for fractional order systems. Commun. Nonlinear Sci. Numer. Simulat. 19(9): 2951-2957. https:/ / doi.org/10.1016/j.cnsns.2014.01.022

[7] Bayram M, Adiguzel HS and Ogrekci S (2015). Oscillation of fractional order functional differential equations with nonlinear damping. Open Phys. 13: 377-382. https://doi.org/10.1515/phys-2015-0053

[8] Bayram M, Adiguzel HS and Secer A (2016). Oscillation criteria for nonlinear fractional differential equation with damping term. Open Phys. 14(1) :119-128. https:/ / doi.org/10.1515/phys-2016-0012

[9] Baleanu D, Mustafa OG and Agarwal RP (2010). Asymptotically linear solutions for some linear fractional differential equations. Abstract Appl. Anal. 2010 Article ID 865139. https://doi.org/10.1155/2010/865139

[10] Bayram M, Secer A and Adiguzel H (2017). On the oscillation of fractional order nonlinear differential equations. Sakarya Ãœniversitesi Fen Bilimleri Enstit $\tilde{\mathrm{A}} \frac{1}{4} \mathrm{~s} \tilde{\mathrm{A}} \frac{1}{4}$ Dergisi 21(6): 1512-1523.

[11] Delavari H, Baleanu D and Sadati J (2012). Stability analysys of Caputo fractional order nonlinear systems revisited. Nonlinear Dyn. 67: 2433-2439. https://doi.org/10.1007/s11071-011-0157-5

[12] Deng W (2010). Smoothness and stability of the solutions for nonlinear fractional differential equations. Nonlinear Anal. 72: 1768-1777.

[13] Duarte Mermoud MA, Aguila Camacho N, Gallegos JA and Castro Linares R (2015). Using general quadratic Lyapunov functions to prove Lyapunov uniform stability for fractional order systems. Commun. Nonlinear. Sci. Numer. Simulat. 22(1-3): 650-659. https://doi.org/10.1016/j.cnsns.2014.10.008

[14] Feng Q (2004). Oscillatory criteria for two fractional differential equations. WSEAS Transactions Mathematics 13: 800-810.

[15] Feng Q and Meng F (2013). Oscillation of solutions to nonlinear forced fractional differential equations. Electronic J. Diff. Equ. 2013(169): 1-10.

[16] Feng Q and Meng F (2013). Interval oscillation criteria for a class of nonlinear fractional differential equations. WSEAS Transactions on Mathematics 12(5): 564-571.

[17] Ganesan V and Kumar SM (2016). Oscillation theorems for fractional order neutral defferential equations. International J. Math. Sci. Engg. Appls. (IJMSEA) 10(III): 23-37.

[18] Nápoles Valdés JE (2021). Oscillatory criteria for some no conformable fractional differential equation with damping. Discontinuity Nonlinearly Complexity, to appear.

[19] Qin H and Zheng B (2013). Oscillation of a Class of Fractional Differential Equations with Damping Term. The Scientific World Journal 685621: 9.

[20] Khalil R, Al Horani M, Yousef A and Sababheh M (2014). A new definition of fractional derivative. J. Computational Appl. Math. 264: 65-70.

[21] Abdeljawad T (2015). On conformable fractional calculus. J. Computational Appl. Math. 279: 57-66. https://doi.org/10.1016/j.cam.2014.10.016 
[22] Baleanu D and Fernandez A (2019). On Fractional Operators and Their Classifications. Mathematics 7: 830. http:/ /dx.doi.org/10.3390/math7090830

[23] Guzmán PM, Langton G, Lugo Motta L, Medina J and Nápoles Valdés JE (2018). A nezw definition of a fractional derivative of local type. J. Math. Anal. 9(2): 88-98.

[24] Fleitas A, Gómez-Aguilar JF, Nápoles Valdés JE, Rodríguez JM and Sigarreta JM (2019). Analysis of the local Drude model involving the generalized fractional derivative. Optik International J. Light Electron Optics 193: 163008.

[25] Fleitas A, Méndez JA, Nápoles Valdés JE and J. M. Sigarreta JM (2019). On fractional Liénard-type systems. Revista Mexicana de Física 65(6): 618-625.

[26] Guzmán PM, Lugo Motta LM and Nápoles Valdés JE (2020). On the stability of solutions of fractional non conformable differential equations. Stud. Univ. Babes-Bolyai Math. 654: 495-502. https:/ / doi.org/10.24193/subbmath.2020.4.02

[27] Guzmán PM and Nápoles Valdés JE (2019). A note on the oscillatory character of some non conformable generalized Liénard system. Adv. Math. Models Applications 4(2): 127-133.

[28] Martínez F and Nápoles Valdés JE (2020). Towards a Non-conformable Fractional Calculus of n-Variables. JMA 43: 87-98.

[29] Nápoles Valdés JE, Guzmán PM and Motta LL (2018). Some new results on the non conformable fractional calculus. Adv. Dyn. sys. Applic. 13(2): 167-175. http:/ /dx.doi.org/10.1155/2013/685621

[30] Nápoles Valdés JE (1998). The historical legacy of the ordinary differential equations. (Self) critical considerations, Boletín de Matemáticas Nueva Serie V: 53-79 (Spanish).

[31] Nápoles Valdés JE (2004). A century of qualitative theory of differential equations. Lecturas Matemáticas 25: 59-111 (Spanish).

[32] Guzmán PM, Lugo Motta Bittencurt LM and Nápoles Valdés JE (2019). A note on stability of certain Liénard fractional equation. International J. Math. Comput. Sci. 14: 301 - 315.

[33] Boudonov N (no dated). "Qualitative theory of ordinary differential equations". Universidad de la Habana, Cuba, (Spanish).

[34] Sansone G and Conti R (1964). "Nonlinear differential equations". MacMillan, New York.

[35] Bekir A, Ã-zkan GÃ $\frac{1}{4}$ ner, Cevikel AC (2013). Fractional Complex Transform and exp-Function Methods for Fractional Differential Equations. Abstract and Applied Analysis 2013 Article ID 426462:8. http:/ /dx.doi.org/10.1155/2013/426462

[36] Li ZB and He JH, (2010). Fractional complex transform for fractional differential equations. Math. Comput. Appl. 15: 970-973.

[37] Mahdy AMS and Marai GMA (2018). Fractional Complex Transform for Solving the Fractional Differential Equations. Global J. Pure Appl. Math. 14(1): 17-37.

[38] Zayed EME, Amer YA and Shohib RMA (2016). The fractional complex transformation for nonlinear fractional partial differential equations in the mathematical physics. JAAUBAS 19: 59-69. https:/ / doi.org/10.1016/j.jaubas.2014.06.008

[39] Jumarie G (2006). Modified Riemann-Liouville derivative and fractional Taylor series of nondifferentiable functions further results. Comput. Math. Appl. 51(9-10): 1367-1376.

[40] Liu CS (2015). Counterexamples on Jumaries two basic fractional calculus formulas. Commun Nonlinear Sci. Numer. Simulat. 22(1-3): 92-94. https:/ / doi.org/10.1016/j.cnsns.2014.07.022 